\title{
PREFERENCIAS EN EL USO DEL TIEMPO LIBRE DE LOS ESTUDIANTES UNIVERSITARIOS CON ENFOQUE EN EL SECTOR TURISMO
}

\author{
Opportunities from the preferences in the leisure time of college students focusing on the \\ tourism cluster
}

\author{
Shirley Bermúdez-Tirado
}

Profesional. Universidad Nacional de Colombia, Medellín-Colombia, sbermudezt@unal.edu.co

\section{Milton Anderson Ramírez-Hoyos}

Profesional. Universidad Nacional de Colombia, Medellín- Colombia, miaramirezho@unal.edu.co

Andrés Jaramillo-Arias

Profesional. Universidad Nacional de Colombia, Medellín- Colombia, andjaramilloari@unal.edu.co

\begin{abstract}
Cómo citar / How to cite
Bermúdez-Tirado, S., Ramírez-Hoyos, M. y Jaramillo-Arias, A. (2016). Preferencias en el uso del tiempo libre de los estudiantes universitarios con enfoque en el sector turismo. Revista CEA, 2(4), 75-88.
\end{abstract}

Recibido: 26 de marzo de 2016

Aceptado: 30 de mayo de 2016

\section{Resumen}

Pocas veces se ha indagado acerca de lo que a un universitario le gusta o le gustaría hacer con su tiempo libre. Por esto se decidió realizar esta investigación, la cual busca conocer cómo los estudiantes de la Facultad de Minas de la Universidad Nacional de Colombia sede Medellín invierten su tiempo libre, identificando preferencias específicas de socialización, salud, diversión, descanso, entre otros componentes que determinan la predisposición a consumir un servicio específico que satisfagan dichas necesidades. Además, se presenta un análisis de los resultados hallados en cuanto a los factores tiempo, dinero, motivación, preferencias e información.

Para desarrollar esta investigación, se diseñaron cuestionarios tipo encuesta y se aplicaron a una muestra de 146 estudiantes de la Facultad de Minas de la Universidad Nacional de Colombia Sede Medellín, con el fin de recolectar información primaria para analizarla usando técnicas de estadística descriptiva.
Se espera que los hallazgos encontrados en este estudio sirvan como insumo para complementar futuras investigaciones sobre el tiempo libre de los estudiantes universitarios.

Palabras clave: tiempo libre, estudiantes universitarios, actividades de ocio, turismo en Antioquia, motivación, preferencias, dinero disponible.

\begin{abstract}
Little has been investigated about what university students like or would like to do with their leisure time. Such is the reason to conduct this research, which seeks to understand how students of the Facultad de Minas of the Universidad Nacional de Colombia - Sede Medellín spend their free time, identifying specific preferences of socialization, health, fun, relaxation, among other components that determine the predisposition to purchase a specific service to satisfy those needs. Moreover, an analysis of the results found in terms of time, money, motivation, preferences and information is presented.
\end{abstract}


To conduct this research, questionnaires, survey type were designed and applied to a sample of 146 students from the Facultad de Minas of the Universidad Nacional de Colombia - Sede Medellín, in order to collect primary data for analysis using descriptive statistical techniques.

It is expected that the findings in this study serve as an input to complement further research on free time of college students.

Keywords: Leisure, free time, college students, tourism in Antioquia, motivation, preferences, money available.

\section{INTRODUCCIÓN}

La investigación permitirá identificar las actividades de tiempo libre que prefieren realizar los estudiantes de la Facultad de Minas de la Universidad Nacional de Colombia - Sede Medellín, profundizando en las actividades turísticas que pueden llevarse a cabo al interior del departamento de Antioquia.

Está demostrado que la mayoría de los estudiantes universitarios disfrutan de actividades extremas como patinaje, skate, autodefensa y experiencias acuáticas (Melton B., Bullet E.; Egli, Bland, Melton \& Czech, 2011), lo que puede ser de utilidad a la hora de promover hábitos saludables. Además, una sana distribución de las actividades que realiza un estudiante en sus períodos de tiempo libre genera sensaciones de bienestar (Huang \& Carleton, 2003).

A pesar de esto, los estudiantes universitarios llevan una vida bastante sedentaria. Se calcula que el $57 \%$ de los hombres estudiantes universitarios, y el $61 \%$ de las mujeres, no realizan el ejercicio físico suficiente; de estos, el $34,38 \%$ comienzan a padecer problemas de salud, como sobrepeso (Buckworth \& Nigg, 2012).

Teniendo en cuenta la problemática planteada, en este proyecto de investigación se abordará y limitará el problema esperando que el planteamiento estructurado y los hallazgos realizados sirvan como base para futuras investigaciones.

\section{MARCO TEÓRICO}

El tiempo libre es todo aquel que tiene una persona luego de cumplir con sus obligaciones y necesidades, es decir, es el resultado de restar el tiempo total que tiene una persona y el tiempo que se utiliza para el trabajo, estudio, familia o relaciones, mientras que el ocio supone sentirse bien realizando determinadas actividades de tiempo libre (Nuviala, 2003), así lo que diferencia estos dos conceptos es que las actividades de ocio suelen realizarse por gusto de la persona. Además, Alcolea, Martori y Sánchez (2003) exponen que las actividades que una persona realiza en su tiempo libre varían dependiendo de las diferentes etapas de la vida. Ahora bien, en términos generales, las actividades de turismo son aquellas que realiza una persona al salir de su entorno habitual y permanecer fuera de este por un período de tiempo inferior a un año (OMT, 1994, citado por Martínez, Pereira, Pawlowska, y López, 2013), si bien, podría haber similitudes entre ocio y turismo, el turismo se caracteriza por ser una expresión social en donde se experimentan nuevas sensaciones caracterizadas por el espacio- tiempo (Gomes y de Souza, 2011).

Profundizando en el concepto de ocio, Dumazedier (1968) y Boullón (1993) expresan que este es el tiempo que las personas tienen disponible después de haber realizado obligaciones laborales, sociales y familiares, el cual pueden ocupar en actividades de distracción y gusto propio, en donde se pueden identificar tres funciones para distribuir dicho tiempo que son: descanso, recreación y desarrollo personal (Román, 2006, citado por Azevedo, Faria, Gomes, Federal y Gerais, 2013).

Realizar actividades de ocio mejora factores esenciales en la vida de las personas, por ejemplo, se encontró que las personas que se 
mostraban más felices que otras utilizaban más su tiempo con amigos y calificaron mejor sus relaciones (Diener, Seligman, 2002, citado en Doerksen, Elavsky, Rebar y Conroy, 2014). Además, quienes disfrutan el ejercicio físico mejoran su bienestar ( Reed y Ones, 2006; Doerksen et al., 2014), ya que este tiene efectos protectores contra la depresión, angustia y ansiedad (Physical Activity Guidelines Advisory Committee, 2008; Doerksen et al., 2014). Pero no solo la actividad física puede generar dichos beneficios, también se ha demostrado que emplear tiempo de ocio en actividades como voluntariado, actividades relacionadas con clubes sociales, actividades religiosas y actividades cognitivas, generan efectos en el bienestar de las personas (Foubert y Grainger, 2006; Doerksen et al., 2014).

Ahora bien, es necesario conocer las implicaciones que el tiempo libre trae sobre las vidas académicas y personales de los jóvenes universitarios. Existen tres elementos básicos para que un estudiante universitario se sienta académicamente satisfecho y no abandone la universidad, los cuales son: actividades académicas, amor propio y participación en actividades extracurriculares (Huang y Carleton, 2003). Se estima que el tiempo de ocio diario promedio de un universitario es de 3,6 horas, que dedica a ver televisión, escuchar música, chatear con los amigos, leer, hacer ejercicio y trabajar en el computador (Honari, Goudarzi y Heidari, 2010). Similar a lo anterior, Bichescu (2014) plantea que el $25 \%$ de los estudiantes tienen de 2 a 4 horas disponibles por día; mientras que el 19\% tienen de 4 a 6 horas; el 15\% de 6 a 8 horas y el $11 \%$ de 8 a 10 horas disponibles. Por otra parte, el $17 \%$ de los estudiantes han afirmado que pueden tener tiempo de ocio cuando lo deseen; $y$ finalmente, el $13 \%$ de los estudiantes, declararon que no tenían tiempo para el ocio (Bichescu, 2014).

Estudios ponen en evidencia que los universitarios pasan su tiempo libre principalmente viendo televisión, con vídeos y usando el computador, por lo que ponen menos atención a la actividad física (Astrid y sjlie 2002, citado en Honari, Goudarzi y Heidari, 2010). Estas actividades se correlacionan positivamente con el desarrollo de problemas psicológicos y enfermedades coronarias (LaMondia, Snell y Chandra, 2009).

Los estudiantes que dedican su tiempo libre a hacer ejercicio informan que el principal factor que influye en su decisión de practicar actividades deportivas es su situación financiera, ya que necesitan dinero para asistir a los gimnasios o para alquilar escenarios deportivos (Bichescu, 2014).

El tiempo que emplean las personas en ocio también puede depender del género, esto se fundamenta en investigaciones que indagan sobre las diferentes motivaciones que sienten hombres y mujer para realizar ejercicio físico, revelando que tienen diferentes motivos para ejercitarse (Egli et al., 2011).

Se calcula que el $57 \%$ de los hombre estudiantes universitarios y el $61 \%$ de las mujeres, no realizan el ejercicio físico suficiente (Buckworth y Nigg, 2012), de estos, el 34,38\% comienza a padecer problemas de salud como sobrepeso, y el peso ganado durante los años de universidad puede incrementar la probabilidad de que un estudiante padezca obesidad en el futuro (Racette, Deusinger, Strube, Highstein, Deusinger, 2011, citado en Egli et al., 2011).

Artinger et al. informaron que la participación de estudiantes universitarios en actividades deportivas genera un impacto significativo en la mejora de su capacidad de trabajar en grupo, y destacó el papel del ocio en la construcción de comunidades más unidas y fuertes (Artinger, Clapham, Hunt, Meigs, Milord, Sampson y Forrester, 2006, citado en Gómez y Glass, 2014).

De los estudiantes que hacen deporte, los hombres prefieren involucrarse en deportes como el fútbol, musculación, natación, voleibol, baloncesto y montañismo; mientras que las mujeres prefieren participar en deportes como 
la natación, el voleibol, escalada, bádminton y tenis de mesa (Honari et al., 2010).

Una de las actividades de tiempo libre preferida por las personas es realizar viajes, y una tendencia que se ha visto en los últimos años es que las personas prefieren viajar al interior de su país o región, de lo cual se resalta que suelen volverse leales a los lugares que más les gustan (LaMondia et al., 2009).

Es sumamente importante resaltar el turismo dentro de este estudio, por lo que evocando varias definiciones se encuentra que Gastal y Moesch (2007) expresan que el turismo involucra un proceso de extrañeza, en donde las personas experimentan un viaje subjetivo e inesperado dependiendo de cada individuo, en el que reavivan recuerdos y experiencias pasadas (Gomes, Ramos y Serejo, 2010, citado por Azevedo et al., 2013). Otros autores lo describen como un intercambio intercultural de un fenómeno humano en el que el individuo se desplaza a lugares fuera del habitual, en donde puede formar diferentes tipos de relaciones (Panosso, 2005, citado por Azevedo et al., 2013). Por lo general, el turismo se puede entender como una relación entre lo social y un desplazamiento, ya sea de viaje, visita o paseo, determinado en un tiempo que generalmente esta fuera de lo laboral y en donde se exhorta al individuo a experimentar no solo experiencias presentes, sino también revivir sentimientos y experiencias pasadas (Cheibub \& Melo, 2010, citado por Azevedo et al., 2013).

El sector turismo tiene un gran impacto en la economía mundial y el desarrollo, ya que al enfocarse en una región determinada promueve la generación de empleos, contribuye con la mejora de la infraestructura, soporta la industria local, incrementa el desarrollo urbano, entre otros aspectos que hacen que las regiones turísticas sean lugares más avanzados. Con el turismo se generan grandes retos, ya que se deben establecer estrategias adecuadas que permitan comprender e identificar las principales características de los viajeros y el por qué eligen cierto destino, generando así servicios que atraigan nuevos turistas (LaMondia et al., 2009).

Los viajes pequeños también forman parte de las actividades que disfruta un joven universitario, aunque el aspecto económico es muy determinante ya que la mayoría no tienen empleo, y esto sumado a que los jóvenes usan frecuentemente las redes sociales, hacen que se vea afectada la elección del destino y el transporte a emplear, mientras que los que usan agencias para planear el viaje no tienden a usar el vehículo personal y planean cada detalle del viaje (LaMondia et al., 2009).

Ahora bien, los acompañantes de un viajero suelen ser determinantes para elegir el lugar a visitar y el medio que se utilizara para viajar. Así, las personas que viajan solas o con niños, prefieren visitar lugares más cercanos a su residencia, mientras que aquellos que viajan acompañados de amigos o pareja prefieren visitar ciudades de gran tamaño, ya que hay más oportunidad de conocer gente y suelen usar su propio vehículo como medio de transporte porque sienten que esto forma parte de la experiencia, permitiéndoles realizar paradas no planeadas (LaMondia et al., 2009).

\section{METODOLOGÍA DE LA INVESTIGACIÓN}

Para desarrollar esta investigación se diseñaron cuestionarios tipo encuesta y se aplicaron a una muestra de 146 estudiantes de la Facultad de Minas de la Universidad Nacional de Colombia Sede Medellín, con el fin de recolectar información primaria para analizarla usando técnicas de estadística descriptiva.

Con estas encuestas, se buscaba obtener la percepción que tienen los estudiantes de la Facultad de Minas de la Universidad Nacional de Colombia, con respecto a las actividades de turismo que pueden realizarse en el departamento de Antioquia, considerando aspectos como el tiempo libre disponible, las diferentes motivaciones, el dinero disponible 
para invertir, los lugares de interés y tipos de actividades a realizar.

Para obtener el número de encuestas a aplicar se realiza un muestreo no probabilístico por conveniencia, donde se realizó una matriz de tamaños muéstrales para diversos márgenes de error y niveles de confianza, dando como resultado, al establecer un nivel de confianza del $95 \%$ y un margen de error del $8 \%$, el total es de 146 encuestas. El tipo de muestreo es no probabilístico y por conveniencias, ya que la población objetivo era muy grande y no se contaba con el tiempo y con los recursos necesarios para realizar un muestreo probabilístico. Se espera que esta investigación sea un punto de partida para realizar estudios más profundos y obtener resultados más precisos sobre la percepción que tienen los estudiantes acerca de las actividades de tiempo libre, ya que a partir de este estudio no es posible dar una conclusión generalizable a todos los estudiantes de la Facultad de Minas de la Universidad Nacional de Colombia. En el análisis, además, se contrastaron los resultados obtenidos con hallazgos en la literatura para aportar elementos a la discusión de la temática de estudio. Para el análisis de la información, se clasificaron los datos obtenidos en la encuesta en cinco factores determinantes para alcanzar los objetivos planteados; los factores son:

- Dinero que tienen disponible los estudiantes para invertir en actividades turísticas

- Tiempo que tienen disponible los estudiantes para invertir en actividades turísticas

- Información que tiene a disposición el estudiante para decidir en cómo invertir su tiempo libre
- Motivación del estudiante para realizar actividades turísticas

- Preferencias del estudiante a la hora de realizar actividades turísticas

\section{ANÁLISIS DE RESULTADOS Y DISCUSIÓN}

Previo al análisis mediante factores es importante presentar el perfil sociodemográfico básico de los estudiantes encuestados, a partir de las preguntas abordadas en el cuestionario para la descripción de dicho perfil. De los 146 estudiantes encuestados 68 de ellos estaban entre los 16 y 20 años; 70 estaban en el rango de los 21 a 25 años; y 8 personas tenían entre 26 y 35. De la población total encuestada, el 30,1\% fueron mujeres; mientras que el 69,9\% fueron hombres. El 39\% de los encuestados está entre primer y quinto semestre; el $54,8 \%$ entre sexto y décimo semestre; y un 6,2\% está en semestres superiores al décimo.

\section{Factor: Dinero que tienen disponible los estudiantes para invertir en actividades turísticas}

En este punto se hace referencia a la cantidad de dinero que tiene a disposición un estudiante de la Facultad de Minas de la Universidad Nacional, que está dispuesto a invertir para realizar actividades de turismo que sean de su agrado.

En la Tabla 1 se puede resaltar cómo la mayor disposición de los estudiantes para elegir los factores que se consideran clave para la realización de actividades turísticas está en invertir sumas de dinero que oscilen entre 39 a 52 dólares, específicamente para los factores actividades nocturnas, recreativas/deportivas, alimentación y alojamiento con porcentajes de $35,20 \%$; $32,63 \% ; \quad 26,37 \%$ y $\quad 40,43 \%$ respectivamente. 
Tabla 1. Dinero a invertir y factores clave

Table 1. Money to invest and key factors

\begin{tabular}{lcccccc}
\hline & \multicolumn{7}{c}{ Dinero a invertir* } \\
\hline & De 8,7 a 13 & De 13 a 26 & De 26 a 39 & De 39 a 52 & De 52 a 65 & $\begin{array}{c}\text { Más de } \\
65\end{array}$ \\
\hline Actividades nocturnas & $0,00 \%$ & $8,00 \%$ & $21,60 \%$ & $35,20 \%$ & $16,00 \%$ & $19,20 \%$ \\
Actividades & $0,30 \%$ & $6,65 \%$ & $21,75 \%$ & $32,63 \%$ & $15,11 \%$ & $23,56 \%$ \\
recreativas/deportivas & $0,00 \%$ & $5,49 \%$ & $26,37 \%$ & $26,37 \%$ & $21,98 \%$ & $19,78 \%$ \\
Alimentación & $0,36 \%$ & $6,50 \%$ & $22,74 \%$ & $40,43 \%$ & $12,64 \%$ & $17,33 \%$ \\
\hline Alojamiento &
\end{tabular}

Fuente: elaboración propia. *Valores expresados en dólares, con base en la TRM de Colombia a 09 de diciembre de 2014.

En cuanto a las actividades recreativas/deportivas y la disponibilidad de dinero a invertir puede realizarse un comparativo con lo propuesto por Bichescu, quien afirma que para los estudiantes que dedican su tiempo libre a hacer ejercicio, el factor que más influye en esto es su situación financiera, ya que necesitan dinero para asistir a los gimnasios o para alquilar campos deportivos (Bichescu, 2014); esto tiene relación con lo encontrado, ya que quienes eligieron el deporte, estarían dispuestos a pagar una mayor cantidad de dinero que quienes eligieron las actividades nocturnas y la alimentación.

Estos resultados implican que un estudiante que busque un lugar donde tenga un buen alojamiento y pueda realizar actividades recreativas y deportivas que sean de su agrado, estaría dispuesto a pagar más dinero que aquellos que prefieren las actividades nocturnas y la buena alimentación.

En el estudio también se observó que el 40,16\% de los estudiantes que quieren realizar una actividad de turismo en Antioquia, y aún no la han podido realizar, expresan que el dinero que tienen disponible para ello es uno de los mayores limitantes, por lo que este tipo de estudiantes se sentiría atraído por planes turísticos donde perciban que no están gastando una gran cantidad de dinero para hacer las actividades de su preferencia.

Finalmente, para este criterio es importante considerar la relación existente entre los medios de transporte planteados y los rangos de disponibilidad a pagar por realizar la actividad de turismo en los municipios de Antioquia, estos se presentan en la Tabla 2.

Tabla 2. Transporte y disposición a pagar

Table 2. Transport and disposition to pay

\begin{tabular}{lcccccc} 
& \multicolumn{7}{c}{ Dinero a invertir* } \\
\cline { 2 - 7 } & De 8,7 a 13 & De 13 a 26 & De 26 a 39 & De 39 a 52 & De 52 a 65 & $\begin{array}{c}\text { Más de } \\
65\end{array}$ \\
\hline Automóvil & $0,96 \%$ & $14,42 \%$ & $24,04 \%$ & $36,54 \%$ & $13,46 \%$ & $10,58 \%$ \\
$\begin{array}{l}\text { Particular } \\
\text { Motocicleta }\end{array}$ & $0,00 \%$ & $18,18 \%$ & $27,27 \%$ & $18,18 \%$ & $9,09 \%$ & $27,27 \%$ \\
$\begin{array}{l}\text { Transporte } \\
\text { público }\end{array}$ & $3,85 \%$ & $7,69 \%$ & $30,77 \%$ & $34,62 \%$ & $11,54 \%$ & $11,54 \%$ \\
\hline
\end{tabular}

Fuente: elaboración propia. *Valores expresados en aproximación a dólares, con base en la TRM de Colombia a 09 de diciembre de 2014. 
De la tabla anterior puede concluirse que un $36,54 \%$ de los encuestados que prefieren el automóvil particular estarían dispuestos a invertir de 39 a 52 dólares en promedio diarios para realizar su actividad, y que un $34,62 \%$ de los que eligieron el transporte público estarían dispuestos a invertir la misma cantidad de dinero para realizar su actividad favorita; estos resultados pueden contrastarse con lo expuesto por Harms et. al., ya que plantean que el modo de transporte es una característica influenciada directamente por el poder adquisitivo (Harms 2006; Grigolon, Kemperman y Timmermans, 2012). Este comentario no se relaciona directamente con los resultados encontrados, ya que un estudiante que use el transporte público estaría dispuesto a invertir la misma cantidad de pesos colombianos por día que quienes viajan en automóvil, aun sabiendo que el primer medio es mucho más económico que el segundo, por lo cual el dinero que se economice un estudiante usando el transporte público podría invertirlo en otros ítems que conformen la realización de la actividad de turismo.
Factor: Tiempo que tienen disponible los estudiantes para invertir en actividades turísticas

El tiempo que tiene disponible los estudiantes encuestados es el factor que afecta directamente las actividades que realiza dicho público, ya que cada actividad tiene diferentes tiempos de duración debido a varios aspectos, tales como el tiempo de viaje hacia el lugar donde se realiza, la preparación previa de la persona para realizarla, la duración misma de la actividad, entre otros, por lo que una actividad con un tiempo de duración mayor requerirá asimismo de una mayor disposición de tiempo libre por parte de los estudiantes.

El $48,57 \%$ de las personas que quieren realizar una actividad de turismo en Antioquia, y aún no la han realizado, expresan que el tiempo que tienen disponible es uno de los mayores limitantes, por lo que este tipo de estudiantes pueden sentirse atraídos por actividades que requieran una menor dedicación de tiempo, ya sea por su corta duración o porque los lugares donde pueden realizarlas están ubicados cerca a su lugar de residencia.

Tabla 3. Número de días por mes que le gustaría salir de la ciudad vs horas libres Table 3. Number of days per month that would like to leave the city vs free hours

\begin{tabular}{ccccc}
\cline { 2 - 5 } & \multicolumn{4}{c}{ Horas libres por semana } \\
\cline { 2 - 5 } & De 0 a 16 & De 17 a 24 & De 24 a 40 & Más de 40 \\
\hline De 1 a 2 días & $9,57 \%$ & $18,09 \%$ & $38,30 \%$ & $34,04 \%$ \\
De 3 a 5 días & $12,17 \%$ & $20,87 \%$ & $28,70 \%$ & $38,26 \%$ \\
De 6 a 8 días & $0,00 \%$ & $36,36 \%$ & $27,27 \%$ & $36,36 \%$ \\
Más de 8 días & $20,00 \%$ & $13,33 \%$ & $40,00 \%$ & $26,67 \%$ \\
\hline
\end{tabular}

Fuente: elaboración propia.

En la Tabla 3, se observa un comportamiento interesante en los extremos, más específicamente se tiene que para los estudiantes con disponibilidad de tiempo de entre 0 y 16 horas libres por semana, el $20 \%$ siendo el porcentaje más alto, les gustaría salir fuera de la ciudad más de 8 días al mes; mientras que aquellos que tienen más de 40 horas libres a la semana estarían menos dispuestos a salir más de 8 días fuera de la ciudad, pero sí se interesan con mayor probabilidad a salir de 3 a 5 días con un $38,26 \%$, lo que se puede interpretar como que un estudiante que cuente con menos tiempo disponible estaría más dispuesto a salir fuera de la ciudad que uno que tenga mayor tiempo libre. Se puede analizar también que las personas que disponen de 17 a 40 horas libres a la semana, tendrían la disposición de salir cada fin de 
semana a visitar diferentes pueblos de Antioquia, con un presupuesto entre $\$ 39$ a $\$ 52$ dólares en promedio, ya que para cada rango de hora la mayoría de los encuestados coincidieron en estar dispuestos a pagar dicho valor diario.

Factor: Información que tiene a disposición el estudiante para decidir cómo invertir su tiempo libre

La información que posee un estudiante de la Facultad de Minas de la Universidad Nacional de Colombia es elemental, no solo para saber qué actividad realizar y dónde realizarla, sino que esta misma información puede llevar a que inicialmente el estudiante se decida a buscar actividades que sean de su agrado, las cuales puedan ayudarlo a contrarrestar las posibles afectaciones que la cotidianidad genera.

El $48,72 \%$ de los estudiantes que quieren realizar una actividad de turismo en Antioquia, y aún no han podido, expresan que la falta de información es uno de los factores que más ha limitado para ellos la realización de las actividades que desean, lo cual implica que poner información suficiente a disposición de estas personas, donde se incluyan actividades, tiempos de viaje, clima, alojamiento, alimentación y costos, podría ser definitivo para facilitar la realización de las actividades de turismo que quieren realizar.

Se analiza la relación entre algunas de las actividades recreativas/deportivas que se planteaban en la encuesta, con los municipios de Antioquia (Colombia) que se caracterizan por realizar dicha actividad, ya sea por sus condiciones geográficas, construcciones o por poseer profesionales expertos en las actividades. Se observa un comportamiento muy particular, ya que las actividades que resultaron ser las más interesantes para el total de los estudiantes encuestados (parapente con un 55,48\% y bungee con un 46,58\%) resultaron ser sobre las que menos se tiene información, ya que los estudiantes encuestados no relacionaron estas actividades con los lugares donde se practican, específicamente, se encontró que los encuestados que expresan estar muy interesados en practicar parapente y bungee son indiferentes o no tienes interés en el lugar caracterizado por realizar dicha actividad; esta falta de información implica una mayor dificultad para tomar la decisión. Por otro lado, actividades como paracaidismo, senderismo y canopy son correctamente asociadas al lugar donde se realizan, ya que los encuestados están interesados en el lugar respectivo a la actividad.

Es importante que ante la desinformación observada se empleen estrategias, ya sea por parte del sector turismo o por parte de las alcaldías municipales, para lograr que las actividades que caracterizan a cada población sean conocidas por un gran número de personas, ya que esto se puede traducir en mayores ingresos para la región. Una de las estrategias puede considerar el uso de las redes sociales como herramienta para transmitir información, ya que el $65,75 \%$ de los encuestados expresaron sentirse influenciados por anuncios turísticos disponibles en Facebook, y un 20,55\% siente gran atracción por anuncios e imágenes en Instagram; este hallazgo es consistente con lo planteado por LaMondia et al. (2009), quienes afirman que los jóvenes usan frecuentemente las redes sociales, y el solo uso de recursos online para planear un viaje, afecta la elección del destino y el transporte a emplear (LaMondia et al., 2009).

El $32,19 \%$ de los estudiantes encuestados expresan tomar decisiones con base a esta información.

\section{Factor: Motivación del estudiante para realizar actividades turísticas}

Este factor se centra en la identificación de elementos que impulsen de manera racional o emocional al estudiante a tomar decisiones alrededor de la realización de actividades de turismo.

De los estudiantes encuestados que desean realizar una actividad de turismo al interior de 
Antioquia, y aún no lo han logrado, el 41,53\% expresó que le gustaría viajar en automóvil particular a los diferentes municipios del departamento; $y$, en segundo lugar, con un $8,47 \%$ usarían el transporte público, lo cual puede traer dos implicaciones. La primera de ellas es que, de tener a disposición un vehículo personal, sería más probable que se sintieran más motivados para realizar las actividades que aún no han realizado; la segunda es que los estudiantes encuestados se sentirían más motivados si se les brindara un servicio que los recoja en la puerta de sus casas, los lleve hacia el lugar de destino y los vuelva a dejar en sus lugares de residencia.

También es importante mencionar que el 37,5\% de los estudiantes que quieren realizar una actividad de turismo en Antioquia, y aún no la han podido realizar, expresan que simplemente no se han sentido motivados para realizar dichas actividades, por lo que deben emplearse estrategias que generen deseo e impulsen al público encuestado a sentir motivación para realizar este tipo de actividades.

Tabla 4. Preferencias y motivaciones para la realización de actividades de tiempo libre según el género Table 4. Preferences and motivations for performing leisure activities by gender

\begin{tabular}{c|cccc|cccc}
\hline \multicolumn{4}{c}{ Preferencias } & \multicolumn{4}{c}{ Motivaciones } \\
\hline Género & Deporte $\begin{array}{c}\text { Ocio en } \\
\text { casa }\end{array}$ & $\begin{array}{c}\text { Salir de } \\
\text { la ciudad }\end{array}$ & $\begin{array}{c}\text { Lugares } \\
\text { comerciales }\end{array}$ & Salud & Diversión & Socialización & Descanso \\
\hline Femenino & $40,91 \%$ & $90,91 \%$ & $50 \%$ & $43,18 \%$ & $25,00 \%$ & $86,36 \%$ & $31,82 \%$ & $79,55 \%$ \\
Masculino & $59,80 \%$ & $72,55 \%$ & $18,63 \%$ & $32,35 \%$ & $29,41 \%$ & $83,33 \%$ & $36,27 \%$ & $66,67 \%$ \\
\hline
\end{tabular}

Fuente: elaboración propia.

Los porcentajes registrados en esta tabla corresponden cada uno de ellos al total de personas que respondieron afirmativamente a la preferencia o motivación indagada.

De la Tabla 4 se obtiene como resultado que el $25 \%$ de las mujeres y el $29,41 \%$ de los hombres encuestados eligieron la salud como factor que los motiva a realizar una actividad de tiempo libre, lo cual indica que los hombres, en comparación con las mujeres, tienen una mayor tendencia a elegir actividades que mejoren su estado físico y los mantenga saludables, tales como el deporte. Este análisis de las motivaciones concuerda con las preferencias relacionadas del deporte, ya que el 59,80\% de los hombres prefieren por encima de las mujeres con un 40,9\% realizar la actividad del deporte.

Aunque estos porcentajes son significativos, no son suficientes para indicar que los estudiantes encuestados realizan actividades de tiempo libre que favorezcan su salud, por lo cual deben emprenderse campañas que motiven a estos jóvenes a dejar su sedentarismo, ya que está demostrado que quienes emplean su tiempo libre en actividades como ejercicio físico, no solo mejoran su bienestar y su salud general (Reed y Ones, 2006; Doerksen et al., 2014), sino que, además, desarrollan cierto efecto protector contra la depresión, la angustia y la ansiedad (Physical Activity Guidelines Advisory Committee, 2008; Doerksen et al., 2014). Por esto, lo deseado sería que más del $50 \%$ de los estudiantes encuestados, tanto hombres como mujeres, sintieran que su salud y bienestar físico y emocional, son razones suficientes para emprender actividades de tiempo libre más activas.

De la Tabla 4, se puede concluir también, que el $86,36 \%$ de las mujeres encuestadas y el $83,33 \%$ de los hombres encuestados, eligieron la diversión como factor que los motiva a realizar una actividad de tiempo libre; esto muestra que ambos géneros realizan aquellas actividades con las que se sienten muy bien emocionalmente, tales como ir a cine, a restaurantes, salir de la ciudad, ver televisión, entre otras.

Además, el 31,82\% de las mujeres y el 36,27\% de los hombres encuestados eligieron la posibilidad 
de socializar como factor que los motiva a realizar una actividad de tiempo libre, lo cual indica que los hombres tienen una mayor tendencia a elegir actividades donde puedan interactuar con otras personas y donde tengan la oportunidad de conseguir nuevas amistades. Con respecto a la última variable, se observa que el $79,55 \%$ de las mujeres y el $66,67 \%$ de los hombres encuestados seleccionaron el descanso como factor que los motiva a realizar una actividad de tiempo libre, lo cual indica que las mujeres tienen una mayor tendencia a elegir actividades donde puedan dormir, meditar, relajarse y estar tranquilas, ya sea mediante ocio en casa, como lo reveló el resultado de las preferencias en este ítem, donde las mujeres eligieron en un $90,91 \%$ hacer ocio en casa por encima de los hombre, quienes registraron un $72,55 \%$. Esto puede relacionarse con los planteamientos de Egli, y con los de LaMondia et al., donde se indica que los jóvenes universitarios hombres dedican más tiempo al ocio en casa en comparación con las mujeres jóvenes universitarias (Egli et al., 2011), contradiciendo lo hallado en esta investigación, donde las mujeres encuestadas superan ampliamente los porcentajes de ocio en casa que presentan los hombres.
También se observó que el $50 \%$ de las mujeres encuestadas, y el $18,63 \%$ de los hombres encuestados, les gusta salir de la ciudad para disfrutar de su tiempo libre. Adicionalmente, el $43,18 \%$ de las mujeres y el $32,35 \%$ de los hombres prefieren visitar lugares comerciales para aprovechar su tiempo libre. Ambos aspectos son coherentes con el hallazgo anterior, donde las mujeres superan a los hombres en cuanto a la motivación por la diversión, por lo que preferirán este tipo de actividades.

\section{Factor: Preferencias del estudiante a la hora de realizar actividades turísticas}

Con este factor se busca reconocer cuáles son las principales preferencias que tienen los estudiantes de la Facultad de Minas de la Universidad Nacional de Colombia - Sede Medellín, al momento de elegir actividades de turismo en Antioquia entre las diferentes ofertas existentes, por ejemplo, cómo prefieren transportarse a los sitios de destino, con qué personas y lo que prefieren realizar al salir.

Tabla 5. Acompañamiento y transporte

Table 5. Accompaniment and transportation

\begin{tabular}{|c|c|c|c|c|}
\hline & & \multicolumn{3}{|c|}{ Preferencia en el acompañamientc } \\
\hline & & Amigos & Pareja & Familia \\
\hline \multirow{3}{*}{$\begin{array}{c}\text { Preferencia en el } \\
\text { modo de viaje }\end{array}$} & Automóvil particular & $76,3 \%$ & $71,8 \%$ & $79,7 \%$ \\
\hline & Motocicleta & $6,8 \%$ & $7,7 \%$ & $6,3 \%$ \\
\hline & Transporte público & $16,9 \%$ & $20,5 \%$ & $13,9 \%$ \\
\hline
\end{tabular}

Fuente: elaboración propia.

Es importante encontrar si existe alguna relación entre el medio de transporte y las personas que acompañan a los encuestados en la realización de las actividades de turismo. Es por esto que en la Tabla 5 se plantea la relación entre estas preferencias, considerando que el 100\% se obtiene al sumar las columnas de los datos, es decir, los diferentes medios de transporte por acompañante. El $79,7 \%$ de los encuestados prefieren viajar con su familia y en automóvil particular, siendo este el resultado más significativo de la tabla y reafirmando resultados anteriores, donde se observó dicha preferencia de los estudiantes encuestados por viajar con su familia. 
Se encontró también que el $71,8 \%$ de los estudiantes encuestados prefieren viajar en automóvil particular con su pareja y el $76,3 \%$ lo prefieren con sus amigos; estos resultados son compatibles con los planteamientos de LaMondia et al. (2009), ya que, según ellos, los acompañantes de un viajero suelen ser determinantes para elegir el medio de transporte. Además, aquellos que viajan acompañados de amigos o pareja prefieren transportarse en su propio vehículo, porque sienten que esto forma parte de la experiencia, ya que pueden realizar paradas no planeadas (LaMondia et al., 2009).

En los tres casos mencionados es factible la generación de estrategias, tales como la gestión de ofertas turísticas donde se incluyan el transporte predilecto y planes según la compañía preferida de los estudiantes encuestados, ya que se observa que son factores de gran influencia. Además, según Besser y Priel habrá mayor disposición a realizar una actividad de turismo si disfrutan de la compañía de otras personas (Besser y Priel, 2006).

Por su parte, puede observarse que las personas encuestadas no disfrutan de la motocicleta como medio para transporte al interior de Antioquia, ni con pareja, ni con amigos y menos con familia, ya que los porcentajes reportados son, respectivamente, $6,8 \% ; 7,7 \%$ y $6,3 \%$.

A continuación, se relacionan el género con la preferencia por los deportes extremos, ya que es necesario saber qué concepción tiene cada género respecto a estos deportes para la generación de estrategias adecuadas. Los resultados obtenidos se encuentran en la Tabla 6.

Tabla 6. Influencias del género en los deportes extremos

Table 6. Gender influences in the extreme sports

\begin{tabular}{cccccccccc}
\hline & \multicolumn{7}{c}{ Preferencia por deportes extremos } \\
\cline { 2 - 8 } & & Parapente & Paracaídas & Bungee & Canopy & Escalado & Kayak & Torrentismo & Rafting \\
\hline \multirow{2}{*}{ Género } & Femenino & $65,9 \%$ & $54,5 \%$ & $45,5 \%$ & $56,8 \%$ & $38,6 \%$ & $27,3 \%$ & $27,3 \%$ & $27,3 \%$ \\
\cline { 2 - 9 } & Masculino & $54,2 \%$ & $58,8 \%$ & $47,1 \%$ & $34,3 \%$ & $33,3 \%$ & $33,3 \%$ & $36,3 \%$ & $40,2 \%$ \\
\hline
\end{tabular}

Fuente: elaboración propia.

Los porcentajes registrados en esta tabla corresponden, cada uno de ellos, al total de personas que respondieron estar muy interesadas en la realización del deporte extremo consultado.

Podemos observar cómo en la Tabla 6, la preferencia por deportes extremos está relativamente distribuida por género, ya que de los 8 deportes extremos que se analizaron, el género masculino tiene mayor preferencia por 5 de ellos, los cuales son: el paracaidismo, el bungee, el kayak, el torrentismo y el rafting. Por su parte, el género femenino tiene mayor preferencia por el parapentismo, el canopy y el escalado. Sin embargo, en los deportes que domina el género femenino no hay una diferencia muy pronunciada con el género masculino, lo cual sugiere desarrollar estrategias para la implementación de planes turísticos que oferten este tipo de actividades para hombres y mujeres.

Esto se contrastará en la Tabla 7 con las demás actividades planteadas en la encuesta, las cuales no se consideran deportes extremos y se busca identificar si el comportamiento es similar a lo visto en el análisis anterior. 
Tabla 7. Género y actividades de turismo de naturaleza de tipo pasivas

Table 7. Gender and nature tourism activities passive type

\begin{tabular}{|c|c|c|c|c|}
\hline & & \multicolumn{3}{|c|}{ Preferencia actividades no extremas } \\
\hline & & Senderismo & Cabalgata & Paintball \\
\hline \multirow{2}{*}{ Género } & Femenino & $25,0 \%$ & $34,1 \%$ & $36,4 \%$ \\
\hline & Masculino & $20,6 \%$ & $21,6 \%$ & $49,0 \%$ \\
\hline
\end{tabular}

Se observa en la Tabla 7 un comportamiento similar al visto con las actividades extremas. Los valores reportados para el género femenino son mayores para dos de las tres actividades analizadas, aunque los porcentajes entre hombres y mujeres reportan valores cercanos.

Este resultado es coherente con el planteamiento de Buckworth y Nigg, quienes exponen que los hombres universitarios dedican una cantidad importante de tiempo a realizar actividades físicas; mientras que las mujeres universitarias están menos dispuestas a realizar estas actividades deportivas exigentes (Buckworth y Nigg, 2012).

Con la información presentada, tanto agencias de turismo como los municipios Antioqueños pueden emprender el diseño de estrategias dirigidas a una población que actualmente no está siendo atendida por las diferentes ofertas turísticas disponibles en el departamento de Antioquia (Colombia), logrando así no solo aumentar directa o indirectamente sus ingresos, sino también aportando a que los jóvenes cambien sus hábitos de sedentarismo y comiencen a realizar otro tipo de actividades que requieran de esfuerzo físico y que satisfagan las necesidades, expectativas y deseos de este público.

\section{CONCLUSIÓN}

Hay una gran oportunidad para las empresas que ofrezcan actividades recreativas y deportivas al interior de Antioquia, en donde los estudiantes, luego de realizar este estudio (enfocado en un grupo de estudiantes de la Facultad de Minas de la Universidad Nacional) han demostrado tener gran interés por realizar actividades tanto recreativas como deportivas en este departamento, y con una disposición a pagar que les permitiría acceder a planes turísticos completos; además se observó un gran deseo por visitar con frecuencia otros municipios Antioqueños. Por el hecho de haber identificado falta de información entre los estudiantes, se genera una gran oportunidad, tanto para empresas del sector turístico como para los mismos municipios, ya que pueden emprenderse campañas creativas para informar, impulsar $\mathrm{y}$ ofrecer paquetes turísticos $\mathrm{y}$ recreativos a este nicho de mercado con base en las actividades y atracciones de cada lugar, que sean de común interés entre los estudiantes.

Se identificó el interés por la realización de deportes extremos, aprovechando las características geográficas del departamento de Antioquia, lo que se puede traducir en un incentivo para que las empresas del sector turismo y los municipios estructuren planes de viaje en los que se incorporen las actividades que resultaron ser de mayor interés, y dado que no hay una diferencia significativa entre géneros, no es necesario dedicar esfuerzos publicitarios para categorizar lugares y actividad según esta variable.

Se contrastaron algunas de las ideas planteadas por los autores referenciados en el marco teórico, se logró apreciar que la selección del 
medio de transporte para desplazarse al interior de Antioquia, no está directamente relacionado con la cantidad de dinero que invertiría un estudiante para realizar la actividad de tiempo libre que disfrute. Además, de las personas encuestadas, los hombres están más dispuestos a realizar ejercicio físico que las mujeres, mientras que las mujeres preferirán aquellas actividades que contribuyan con su descanso y relajación.

\section{REFERENCIAS}

Alcolea, I.; Martori, E. y Sánchez, C. (2003). Organizaciones y actividades en Cataluña, 2(1), 25-33.

Azevedo, J.; Faria, S.; Gomes, C. L.; Federal, U. y Gerais, D. M. (2013). El ocio y el turismo en los artículos publicados en revistas académicas de turismo. Estudios y Perspectivas en Turismo, 22, 875-892. Retrieved from Recuperado de http://www.redalyc.org/articulo.oa?id=180 728713004

Besser, A. y Priel, B. (2006). Models of attachment, confirmation of positive affective expectations, and satisfaction with vacation activities: A pre-post panel design study of leisure. Personality and Individual Differences, 41(6), 1055-1065. http://doi.org/10.1016/j.paid.2006.04.011

Bichescu, A. (2014). Leisure Time Sport Activities of the Students at Resita University. Procedia - Social and Behavioral Sciences, 117, 735740.

http://doi.org/10.1016/j.sbspro.2014.02.29 1

Buckworth, J. y Nigg, C. (2012). Physical activity, exercise, and sedentary behavior in college students. Journal of American College Health: J of $A C H$, 53(1), 28-34. http://doi.org/10.3200/JACH.53.1.28-34
Doerksen, S. E.; Elavsky, S.; Rebar, A. L. y Conroy, D. E. (2014). Weekly Fluctuations in College Student Leisure Activities and Well-Being. Leisure Sciences, 36(1), 14-34. http://doi.org/10.1080/01490400.2014.860 778

Egli, T.; Bland, H. W.; Melton, B. F. y Czech, D. R. (2011). Influence of age, sex, and race on college students' exercise motivation of physical activity. Journal of American College Health: J of ACH, 59(5), 399-406. http://doi.org/10.1080/07448481.2010.513 074

Gomes, C. y de Souza, T. R. (2011). La temática del ocio según los docentes de las carreras de turismo. Minas Gerais, Brasil. Estudios y Perspectivas en Turismo, 20(1), 127-148.

Gómez, E. y Glass, C. R. (2014). International Student Adjustment to College: Social Networks, Acculturation, and Leisure. International Student Adjustment to College: Social Networks, Acculturation, and Leisure, 32(1), 7-25. Retrieved from http://eds.b.ebscohost.com.ezproxy.unal.ed u.co/eds/detail/detail?vid=3\&sid=50c641bf1cd9-4887-b7c5dd07c9935679@sessionmgr110\&hid=116\& bdata=Jmxhbmc9ZXMmc2|OZT1/ZHMtbG|2Z $\mathrm{Q}==$

Grigolon, A. B.; Kemperman, A. D. a M. Y Timmermans, H. J. P. (2012). The influence of low-fare airlines on vacation choices of students: Results of a stated portfolio choice experiment. Tourism Management, 33(5), 1174-1184.

http://doi.org/10.1016/j.tourman.2011.11.0 13

Honari, H.; Goudarzi, M. y Heidari, A. (2010). An investigation of the ways Tehran university students follow a specific life style and pass their leisure time with an emphasis on sport. Procedia - Social and Behavioral Sciences, 2(2), 5498-5501. 
http://doi.org/10.1016/j.sbspro.2010.03.89 6

Huang, C. y Carleton, B. (2003). The relationships among leisure participation, leisure satisfaction and life satisfaction of college students in Taiwan, 1, 129-132.

LaMondia, J.; Snell, T. y Chandra, B. (2009). Traveler Behavior and Values Analysis in the Context of Vacation Destination and Travel Mode Choices: A European Union Case Study. The University of Texas at Austin (November 2009), 23.
Martínez, F.; Pereira López, X.; Pawlowska, E.; Roget, F. M. y López, X. P. (2013). El turismo académico en Galicia: otra forma de contribución de las universidades a las economías locales. Cuadernos de Turismo, $32,229-242$.

Nuviala, A. (2003). Tiempo libre, ocio y actividad física en los adolescentes. La influencia de los padres. Retos. Nuevas Tendencias en Educación Física, Deporte y Recreación, 1320. 